\title{
Modelling Inductive Charging of Battery Electric Vehicles using an Agent-Based Approach
}

\author{
Zain Ul Abedin ${ }^{* 1}$, Rashid Ahmed Waraich ${ }^{2}$ \\ ${ }^{1}$ Department of Transportation and Traffic Engineering \\ TUM CREATE, Singapore, Singapore \\ e-mail: zain.ulabedin@ tum-create.edu.sg \\ ${ }^{2}$ Institute for Transport Planning and Systems \\ ETH Zurich, Switzerland
}

Cite as: Ul Abedin, Z., Waraich, R. A., Modelling Inductive Charging of Battery Electric Vehicles using an Agent-Based Approach, J. sustain. dev. energy water environ. syst., 2(3), pp 219-233, 2014, DOI: http://dx.doi.org/10.13044/i.sdewes.2014.02.0018

\begin{abstract}
The introduction of battery electric vehicles (BEVs) could help to reduce dependence on fossil fuels and emissions from transportation and as such increase energy security and foster sustainable use of energy resources. However a major barrier to the introduction of BEVs is their limited battery capacity and long charging durations. To address these issues of BEVs several solutions are proposed such as battery swapping and fast charging stations. However apart from these stationary modes of charging, recently a new mode of charging has been introduced which is called inductive charging. This allows charging of BEVs as they drive along roads without the need of plugs, using induction. But it is unclear, if and how such technology could be utilized best. In order to investigate the possible impact of the introduction of such inductive charging infrastructure, its potential and its optimal placement, a framework for simulating BEVs using a multi-agent transport simulation was used. This framework was extended by an inductive charging module and initial test runs were performed. In this paper we present the simulation results of these preliminary tests together with analysis which suggests that battery sizes of BEVs could be reduced even if inductive charging technology is implemented only at a small number of high traffic volume links. The paper also demonstrates that our model can effectively support policy and decision making for deploying inductive charging infrastructure.
\end{abstract}

\section{KEYWORDS}

Inductive charging, Battery electric vehicles, Agent-based modelling.

\section{INTRODUCTION}

Fossil energy carriers are currently the primary source of energy in the world, constituting ca. $80 \%$ of total energy supply [1]. The transportation sector is almost entirely dependent on fossil fuels and is responsible for ca. $20 \%$ of total $\mathrm{CO}_{2}$ emissions globally [2]. Due to decreasing reserves of fossil fuels and possible implications of climate change, an alternative source of energy is required for the transportation sector. One option to reduce the dependence on fossil fuels could be a shift to battery electric vehicles (BEVs) which do not only drive more efficiently than internal combustion engine (ICE) vehicles, but also the electricity required can come from various sources, including renewable ones such as solar, wind and hydro power.

However there are also certain limitations associated with BEVs such as their high initial cost, range anxiety and long charging durations [3]. The current cost of a

\footnotetext{
* Corresponding author
} 
lithium-ion battery for usage in a BEV is 500-650 US\$/kWh [4]. By assuming an average consumption of $0.2 \mathrm{kWh} / \mathrm{km}$ a BEV with a range of $120 \mathrm{~km}$ requires $24 \mathrm{kWh}$ battery which costs 12,000 US\$. In terms of range, a typical BEV can travel ca. $160 \mathrm{~km}$ on fully charged battery whereas an ICE can travel ca. $500 \mathrm{~km}$ on a full tank [5]. One can charge a BEV with a battery size of $24 \mathrm{kWh}$ in ca. 7 hours based upon a power output of $3.3 \mathrm{~kW}$. In contrast to this an ICE vehicle can be refuelled in a few minutes thus making it is far more flexible.

There are possible alternative charging methods available to allow rapid charging, such as battery swapping, fast charging and inductive charging. Battery swapping can help to recharge the battery in just a few minutes by swapping the depleted battery with a fully charged battery. But it requires an extensive network of battery swapping stations and also standardization is required for batteries, vehicles and swapping technology [6]. With fast charging, meaning charging a vehicle with higher power, a battery of $24 \mathrm{kWh}$ can be charged in less than $30 \mathrm{~min}$ with a power output of $60 \mathrm{~kW}$. The cost of such fast charging station is expensive, according to [7] the total cost can lie between 65,000-70,000 US\$ per charging station therefore it is not suitable for BEV users at home, but could be an option at public charging points. Inductive charging can be installed stationary as a substitute for plugs or along the roads [8], where a BEV can charge while driving. It is implemented by embedding inductive charging equipment below the road surface, where a vehicle driving over it can charge. Such inductive charging infrastructure along roads could act complementary to stationary charging at home and public places.

Since the cost of such infrastructure is relatively expensive, a methodology is required to understand the optimal location for electrified links and to investigate the impacts of this technology on BEV charging behaviours and battery size.

In this paper an electric vehicle (EV) simulation framework is used, which itself is based on an agent based transport simulation. Moreover, the extension of the framework by an inductive charging module is described, together with initial simulation results and analysis of BEV charging behaviour. The rest of the paper is organized as follows: The next section presents related work and basic knowledge in this field. In section methodology the inductive module, influencing parameters and assumptions are explained. Section simulations describe the simulation scenarios and results, which are discussed in the discussion and future work section along with some outlook about future work. The paper ends with a conclusion section.

\section{BACKGROUND AND RELATED WORK}

Various researchers around the world are working on inductive charging technology. Only recent work with focus on inductive charging of electric vehicles is presented in this section. Due to the novelty of this technology only little literature is present at the moment.

\section{Real World Application}

The Korean Advance Institute of Science and Technology has developed and utilized shaped magnetic field in resonance to charge BEVs dynamically [9]. Furthermore, they implemented this technology for electric buses which are in operation at their university campus.

Kobayashi, et al. [10] developed and experimented with inductive charging technology with a micro electric bus. The bus has a route of $5.24 \mathrm{~km}$ and it requires 9 minutes of charging duration to complete its route. Thus, the microbus can continuously run on such a cycle, without stationary charging. 


\section{Simulation Based Approach}

Mohrehkesh and Nadeem [11], who analyse different strategies for charging BEVs inductively during stoppage time at intersections. Jang, et al. [12] used a genetic algorithm to optimize inductive charging locations and battery size for battery electric buses on a circular route.

According to the best of the authors' knowledge, we are the first to simulate inductive charging using an agent based approach to evaluate the potential of this technology. In the following sections, we provide information on the traffic simulation and the electric vehicle simulation framework, on which our work is based upon. Thereafter a short description of inductive charging is given to provide background information regarding this technology.

\section{Multi Agent Transportation Simulation (MATSim)}

MATSim [13] is an agent-based travel demand simulation used to simulate large scale traffic scenarios (see Figure 1). In MATSim each agent has a set of plans for daily activities such as work, leisure, shopping and education. Each plan is executed and scored according to a utility function. Performing an activity increases utility whereas travelling gives negative utility. The goal of each agent in MATSim is to maximize its utility. When the plans of agents are executed in the micro-simulation, traffic is generated on a virtual network and thus may lead to congestion. In order to avoid congestion and improve utility, agents can change their plans after each iteration within a replanning step. Changes to the plan can be such as changing the route, travel mode, travel time, activity duration and location. Each agent maintains a set of plans, from which either an old plan is reselected for execution or a new plan is generated. As the set of plans is of limited size, the plan with the worst score is removed when creating a new plan. This corresponds to mutation, selection and survival of the fittest using a co-evolutionary algorithm [14]. Through this process the demand is optimized iteratively.

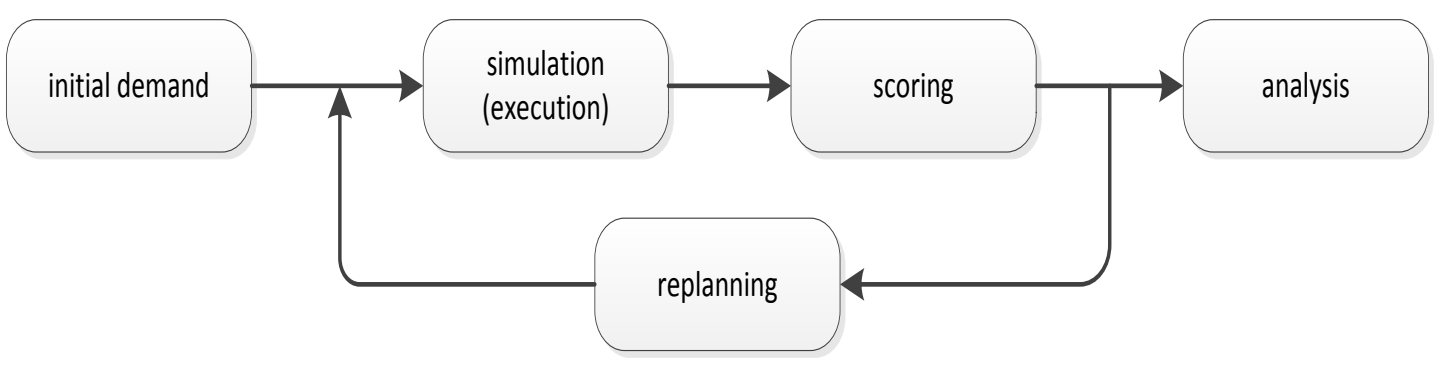

Figure 1. Building blocks of MATSim

Initial demand. It contains initial plans of the agents; a typical plan of an agent consists of activities such as home, work and leisure with their location and time duration.

Execution. It handles the mobility simulation by taking the plans of the agents into account and then runs it over a virtual network while respecting the physical boundaries of a real network. The simulation used in MATSim is based on a queue based traffic model which is capable of representing basic traffic situation such as traffic jams and tailbacks.

Scoring. It assigns scores to each of the performed plan after execution of the mobility simulation MATSim uses a utility based approach which is adopted from 
Vickery bottle neck model [15] and altered to be consistent with the complete daily plans approach [16-18]. The score of a plan is the sum of utilities and dis-utilities earned through performing activities, late arrivals and travelling time. The total utility is calculated according to:

$$
U_{\text {total }}=\sum_{i=1}^{n} U_{\text {perf }, i}+\sum_{i=1}^{n} U_{\text {late }, i}+\sum_{i=1}^{n} U_{\text {travel }, i}
$$

where $U_{\text {total }}$ is the combined total utility for a given plan; $n$ is number of activities; $U_{\text {perf }, i}$ is the positive utility gained by performing an activity $i, U_{\text {late }, i}$ is the negative utility gained for late arrival at an activity i, $U_{\text {travel }, i^{i}}$ is the negative utility gained for travelling during the trip $i$.

The positive utility gained while performing an activity is given as:

$$
U_{\text {perf }, i}\left(t_{\text {perf }, i}\right)=\beta_{\text {perf }} \times t_{*, i} \times \ln \left(\frac{t_{\text {perf }, i}}{t_{0, i}}\right)
$$

where $t_{\text {perf,i }}$ is the actual performed time period of an activity $i, t_{s, i}$ is the usual time period of an activity $i, \beta_{\text {perf }}$ is the marginal utility of an activity during its usual duration and it is same for all activities, as in equilibrium all activities at their usual time period need to have similar marginal utility. The scaling parameter $t_{0, i}$ is related to both the minimum time period and the importance of an activity.

The negative utility gained for late arrival at an activity is given as:

$$
U_{\text {late }, i}=\beta_{\text {late }} \times t_{\text {late }, i}
$$

where $\beta_{\text {late }}$ is the marginal utility for arriving late which is generally negative and $t_{\text {late }, i}$ is the number of hours late at an activity $i$.

The negative utility for travelling is calculated as:

$$
U_{t r_{,} i}=\beta_{t r} \times t_{t r, i}
$$

where $\beta_{t r}$ is the marginal utility for traveling which is also negative and $t_{t r, i}$ is the number of traveling hours consumed during trip $i$.

Replanning. It modifies the plans of the agents, e.g. route, mode of transport, activity duration or departure time change. As the number of plans which the agent can keep is limited due to memory limitations (e.g. to 5), whenever a new plan is created, the worst plan is removed from memory. This new plan is then executed again and scored as part of the iteration loop in MATSim. This iteration process continues until no agent can improve its score significantly.

Analysis. When the system has relaxed (no significant improvement of score per iteration), the results are analysed.

\section{Transport Energy Simulation Framework (TES)}

In our work, we used an EV simulation framework, which builds on top of MATSim [15]. This framework allows modelling a wide variety of scenarios for electric vehicle 
simulation, ranging from smart grid over vehicle to grid to model the effect of charging on power grids [19-21].

One of the reasons for choosing this framework is that it is open source and allows reusing modules implemented by other researchers. Also, its modular design facilities extension and customization. We skip a more detailed description of the framework here, as we will discuss it in the methodology chapter, how the inductive charging module was integrated into this framework.

\section{Inductive Power Transmission}

In this section more information regarding inductive power transmission is provided, which is required to build an inductive charging infrastructure along roads. A typical inductive power transmission between transmitter and receiver coil is shown in Figure 2. The transmitter coil L1 and receiver coil L2 form a system of magnetically coupled inductors. When the alternating current flows in the transmitter coil, a magnetic field is generated which induces a voltage in the receiver coil. This voltage can be used to power any electronic device or battery. The efficiency depends upon the distance between the coils $\mathrm{Z}$ and the relative size of the coils D2/D1 [22].

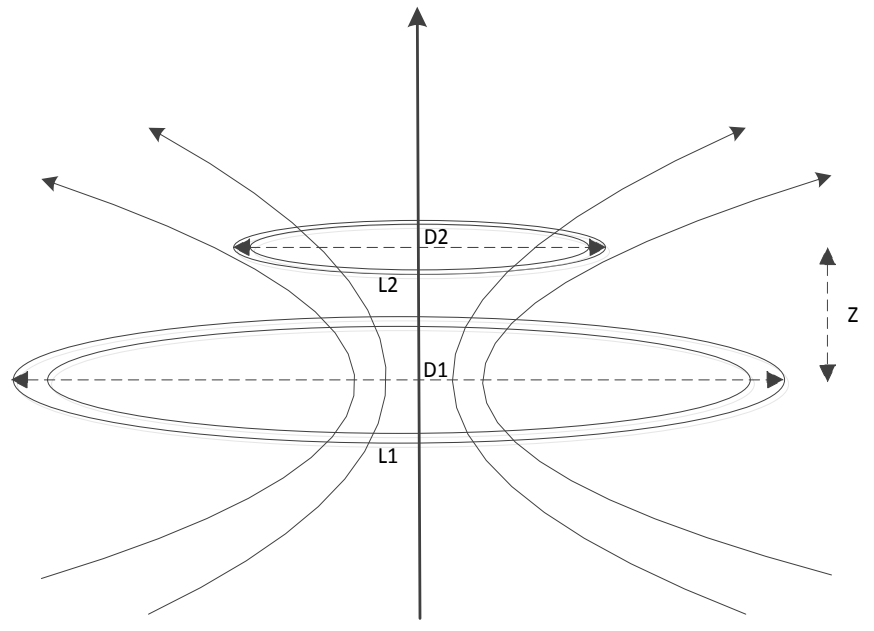

Figure 2. Typical arrangement of inductive power transfer system

When inductive technology is used for charging, the power flows via magnetic coupling between the power source unit generally buried under the ground surface and the receiver unit which is installed under the vehicle. If several power source units are present adjacent to each other, continuous power can be transferred to the receiver unit by simply moving it on top of power source units. This is called dynamic inductive charging, which is abbreviated as inductive charging in this paper. However, there are several design parameters which influence the inductive charging such as power, efficiency, vertical clearance, lateral displacement, electromagnetic field levels and economic viability $[8,23]$. For practical reasons only certain values for these parameters are suitable, which are explained below.

Power. The output power should be comparable to that of fast charging station, e.g. above $25 \mathrm{~kW}$ for BEV car, such that it is practical.

Efficiency. The power transfer efficiency should be high enough so that it can be comparable to that of a charging station which is generally $90 \%$. 
Vertical clearance. The BEV should be able to charge, while keeping a minimum distance to the ground. A vertical gap of $16 \mathrm{~cm}$ between the road surface and vehicle bottom is fair enough for smooth driving.

Lateral displacement. As unlike trains moving on rails, vehicles on roads mostly do not follow a straight path, a tolerance of $30 \mathrm{~cm}$ for horizontal movement is also required, such that even if sending and receiving inductive unit are not inline, still charging is possible.

Electromagnetic field levels. Electromagnetic field shielding should be provided to minimize the electromagnetic field exposure to users.

Economic viability. Economic aspects of such a solution are also important, especially for wide scale implementation. At present not many cost estimates of such an inductive charging system are available, but according to IEEE SPECTRUM [24] the cost for installation of such a system in existing asphalt road may cost ca. $1.5 \mathrm{mln}$ US\$ per lane per $\mathrm{km}$.

\section{METHODOLOGY}

As mentioned in the background section, we have extended a framework for simulation of electric vehicle scenarios, in order to simulate inductive charging along roads. The Transportation Energy Simulation (TES) framework itself contains several interfaces and modules, to simulate a variety of scenarios. Figure 3 shows an overview of the framework, which uses the MATSim simulation. The framework plugs into the MATSim simulation framework at different points:

- This is the point, where TES framework can perform one time initialization operations, such as defining at which parking/road charging infrastructure should be available (0);

- This point is just before a new iteration in MATSim starts, but already part of the MATSim iteration loop. Here operations, which are part of the optimization, can take place, e.g. a policy change (1);

- During execution of the MATSim simulation, TES simulates the energy consumption and charging of vehicles, according to the scenario setup (2);

- After the execution of the micro-simulation in MATSim, the TES simulation produces output for further analysis and also updates the score, e.g. related to charging cost. As such the decisions related to electric mobility of an agent can influence other travel decisions of agents (3);

- During replanning the plan of the agent can be adapted. For example, people having easier access to charging stations might prefer BEVs, while others might prefer plug-in hybrid electric vehicles (PHEVs) or switch to a different mode. Also the route can be chosen differently by different vehicles, e.g. lane reserved for BEV providing inductive charging (4).

For integration of the inductive charging module, the initialization of electrified roads is done at step (0). The charging of vehicles happens at point (2). The state of charge (SOC) of the electric vehicles is updated on a per link basis, as the MATSim traffic model does not support any dynamics or interaction of vehicles inside roads. This means, the SOC of the vehicles is updated after the vehicles have travelled along a road, considering both the energy consumption and energy charged. At the moment, for all vehicles only one type of power is available, which should change in future, see future work section. 


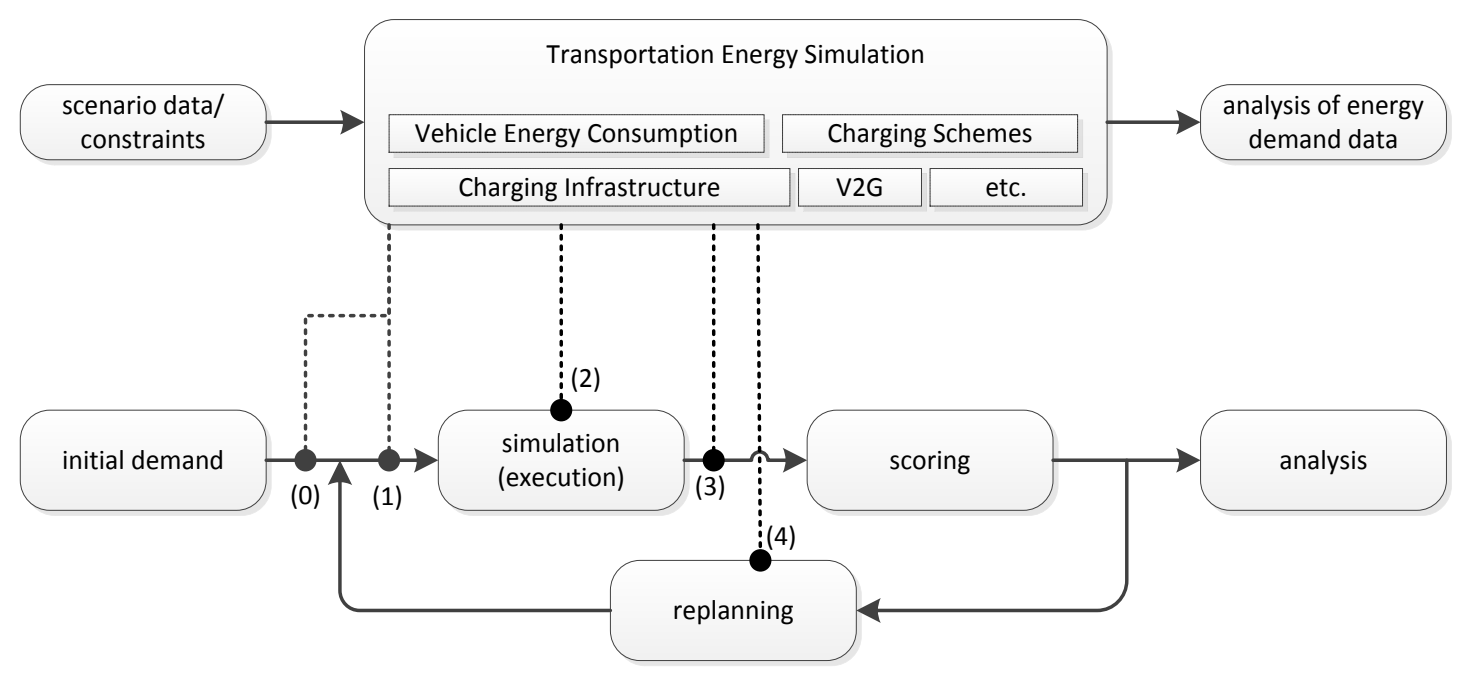

Figure 3. Interfacing of MATSim with TES

At the time we conducted this work, only a limited set of energy consumption models were available for BEVs in TES. We contributed an energy consumption model of electric vehicles to the TES framework, which is adopted from the work of Faria, et al. [25] and is shown in Figure 4. This energy consumption model is based upon the specification of Nissan Leaf BEV. In TES the energy consumption model act as a lookup table which is composed of paired values of energy required at different speed levels.

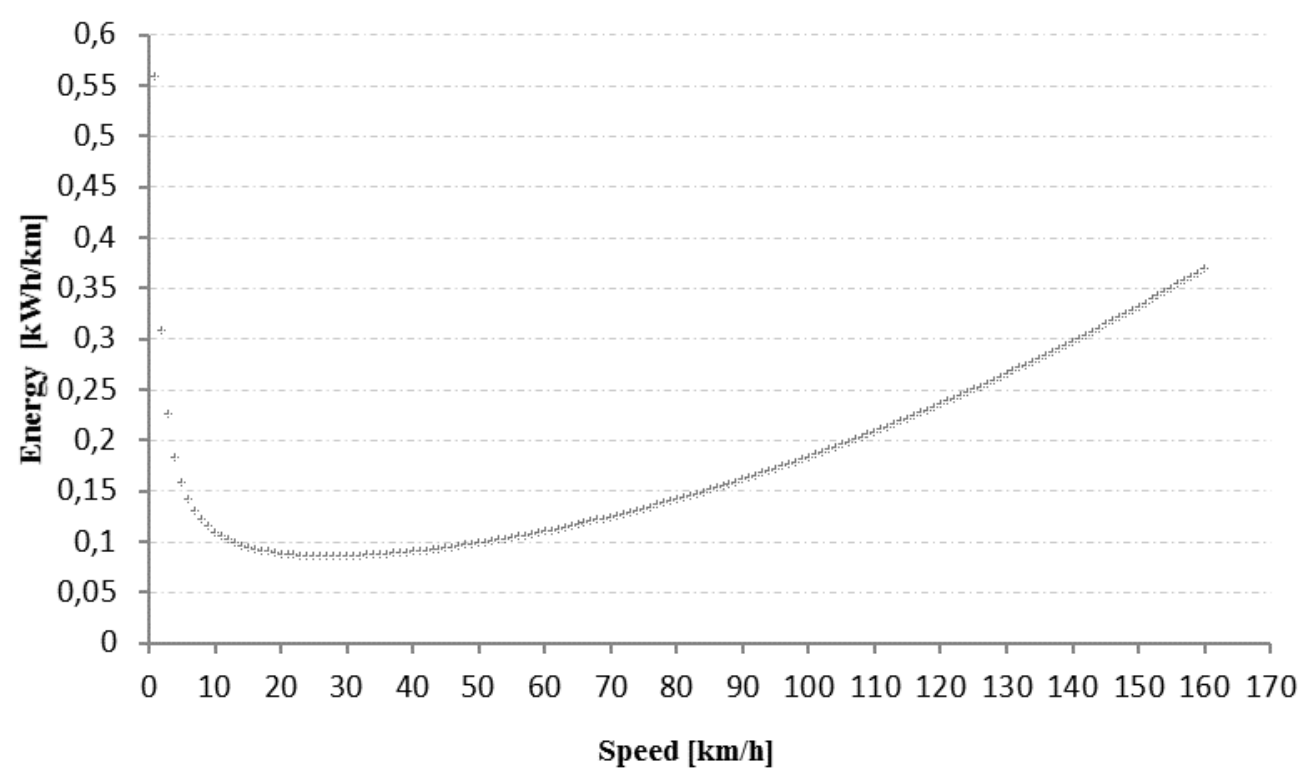

Figure 4. Energy consumption model of BEV

Figure 5 exemplifies how the SOCs of three agents develop as they drive on a road network where only ca. $2 \%$ of the roads are inductive charging enabled (power output of $10.65 \mathrm{~kW}$ and battery size of $11 \mathrm{kWh}$ ). One can observe that the SOC of two agents i.e. agent 0 at link 8 and agent 65 at link 4 has increased due to inductive charging. In contrast, agent 33 didn't find any inductive charging enabled links on its route therefore its SOC never increases. 


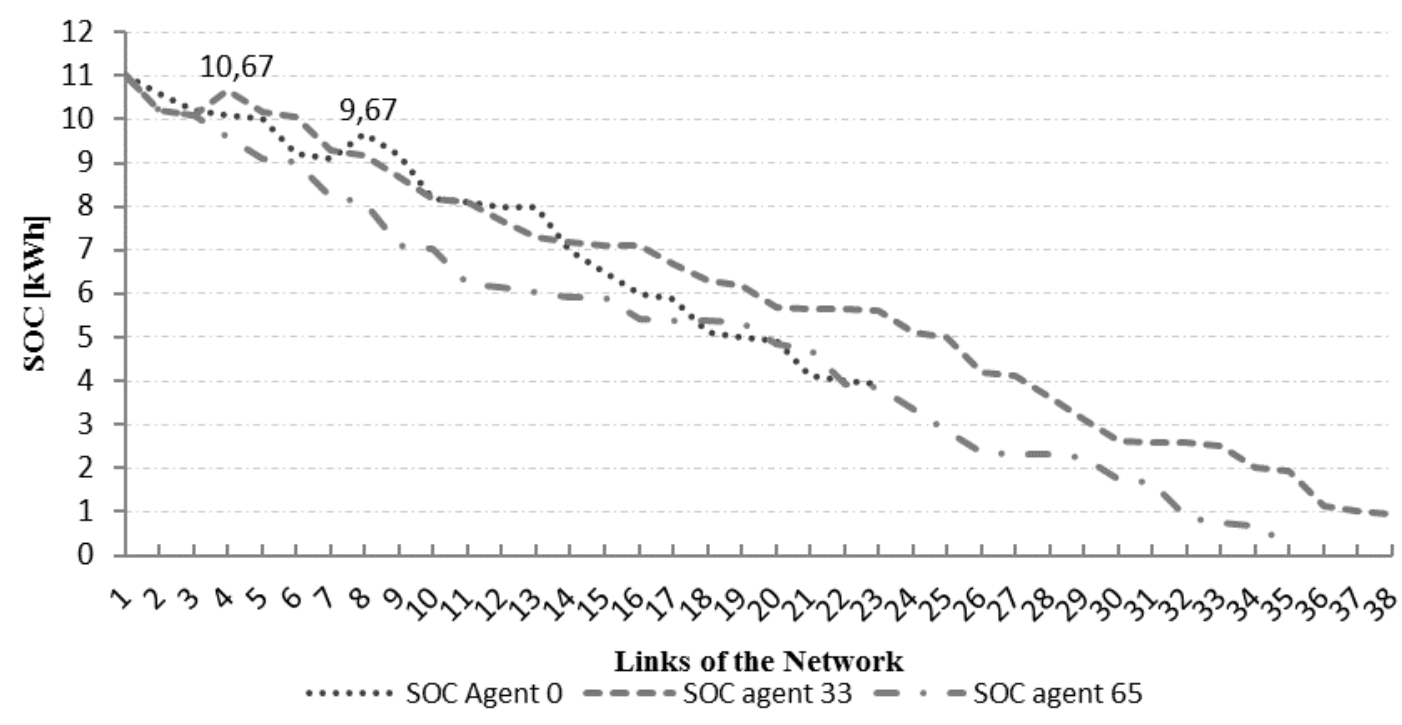

Figure 5. Three selected agents with in the network their SOC

\section{Parameters}

Inductive charging scenarios are tested by enabling only some links with inductive charging. Several parameters influence the charging, which are described in the following.

The charging power can influence inductive charging behaviour of BEVs. More power can be transferred inductively depending upon the power available at the links and vehicle receiver coil capabilities. As the surface at the bottom of BEVs is limited, the available power is also limited. A power of $15 \mathrm{~kW}$ is used which is based on empirical data of inductive charging for a sports utility vehicle (SUV) [23].

Also the length of the electric link plays an important role for inductive charging. The longer the link size, the higher the potential of inductive charging. However, the cost of these electrified roads is much higher than for normal roads therefore the length of these links should be considered during optimization or only parts of roads should be enabled for inductive charging, as discussed in the future work section.

Location of these electrified links is also an important parameter. In large networks it becomes more complicated to find suitable locations. One such location could be at the intersection (see [11]) or high traffic count links. Also congested links are good candidates, as the possibility to charge depends also on the time the BEV spends on such inductive charging links.

Related to this is the maximum allowed speed for driving on an electrified road, as it plays an important role on how much energy can be charged on a link. Lower speed links allow for more charging per meter, but on the other side they are less attractive due to longer travel time.

\section{Assumptions}

Network. An abstract grid based urban road network was developed, which has a total length of $483 \mathrm{~km}$. There are two versions of the network; the first one is used for first two scenarios whereas the second is used for third scenario. The first version of network contains 62 nodes and 140 links whereas the second version contains 110 nodes/intersections and 236 links/roads. Although both versions have different number of nodes and links but the total length and topology of the network is similar. The 
network properties include the free flow speed, flow capacity, no of lanes and modes of transport. The first version of the network in depicted in Figure 6.

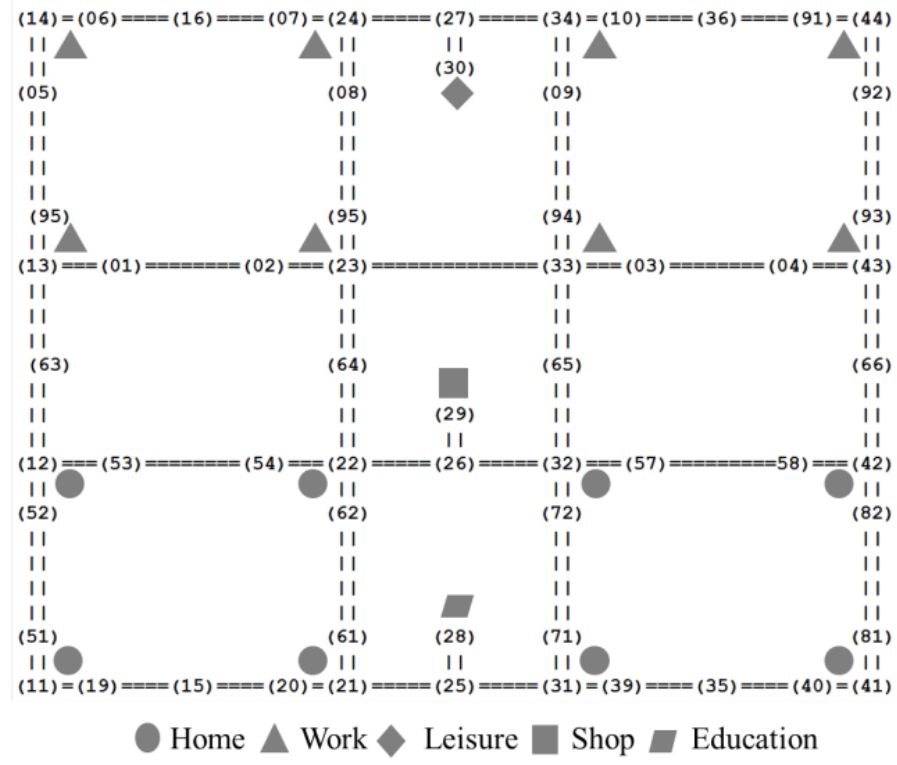

Figure 6. Abstract network and facility distribution

Facilities. In the above mentioned network facilities such as home (h), work (w), leisure (1), shop (s) and education (e) are located as depicted in Figure 6.

Plans of the population. 100 agents are simulated among which 34 have activities chain h-w-h, 22 have h-w-1-h, 14 have h-w-l-s-h, 10 have h-e-h, 10 have h-e-l-h, 5 have h-s-h and 5 have h-s-l-h.

One example of an activity chain is h-w-l-h: an agent leaves home at 8:00 am in the morning for work. It drives the vehicle to its workplace and reaches it at 8:30 am. The agent works for 08:30 hours and then leaves its workplace at 05:00 pm and drives its vehicle to a leisure facility. It reaches the facility at 5:30 pm and does sports for 01:30 hours then it leaves the leisure facility at 7:00 pm and finally reached home at 8:25 pm. The activity chains of the other agents are accordingly.

Vehicles. Identical BEVs are used with same battery size, which is defined per simulation scenario.

Inductive power. The power level used for inductive charging is $15 \mathrm{~kW}$ with $71 \%$ efficiency i.e. $10.65 \mathrm{~kW}$.

\section{SIMULATIONS}

Initially the simulation is run for 100 iterations and it is observed that it reaches its relaxed state after 45 to 50 iterations. A relax state is a state when all the agents optimize their daily plans and no further improvements is possible in their plans, without other agents changing their plan. Therefore the $50^{\text {th }}$ iteration is used as an input for TES to analyse inductive charging. The main parameters that are varied during the first two simulation scenarios are electrification of the links and stepwise battery size reduction. 


\section{Electrification of network based upon high occupancy traffic links}

Electrification of network. In this first experiment the electrification rate (inductive charging) of the network is incrementally increased. The traffic counts are calculated from the analysis process of MATSim and then these are sorted according to the descending order. Only those links which have the highest traffic count are equipped with inductive charging. This is calculated based on the output of the $50^{\text {th }}$ iteration of the traffic simulation. In each electrification step ca. $2 \%(5 \mathrm{~km})$ network links with the highest traffic count are selected for inductive charging, which have not been electrified yet.

Speed. The maximum speed that BEVs can achieve at all links is set to $50 \mathrm{~km} / \mathrm{h}$. The actual speed driven depends on street capacity constraints and interaction between vehicles.

Capacity of the links. In this experiment the capacity of the links is 2,000 vehicles per hour per lane. Since only 100 vehicles are simulated in the experiments which is far less than the capacity of links. Therefore the majority of vehicles drive at a constant speed of $50 \mathrm{~km} / \mathrm{h}$ (maximum speed).

Stepwise battery reduction. The initial battery size of all BEVs is chosen such that the agent who travels the maximum distance during the day is able to complete its trip. Thereafter a stepwise reduction of $1 \mathrm{kWh}$ in battery size is performed. With a reduced battery size, the simulation is run again and analysis is performed regarding how many BEVs run out of battery during driving. Afterwards, the simulation is run again with electrified links of $5 \mathrm{~km}$ length which corresponds to ca. $2 \%$ of the network size. Impact of electrification on those agents who run out of battery is analysed, and checked how many of them took advantage of such electrified segments. Then the percentage of electrification of the network is increased by same step size until all the agents perform their plans without running out of the battery.

For instance, as shown in Figure 7 when the battery size is reduced from $12.5 \mathrm{kWh}$ to $11.5 \mathrm{kWh}, 10 \%$ of the agents were unable to perform their plans, with no electrified links. Later when ca. $2 \%$ of the network is electrified the number of agents who were unable to perform their plans drops from $10 \%$ to $2 \%$. When the electrification is raised from ca. $2 \%$ to ca. $4 \%$, all the agents are able to complete their plans.

From the simulations it is clear that the size of the battery can be reduced significantly provided that certain percentage of the network links is electrified. The battery size can be reduced from $12.5 \mathrm{kWh}$ to $7.5 \mathrm{kWh}$ by electrifying $17 \%$ of the total network links and this reduction accounts for $40 \%$ of battery size reduction.

According to initial tests, $90 \%$ agents can be satisfied in case of $9.5 \mathrm{kWh}$ battery by electrifying ca. $6 \%$ of the network links. This accounts for a battery size which is ca. $75 \%$ of its original size $(12.5 \mathrm{kWh})$.

\section{Extension of electrification of network based upon high occupancy traffic links, with congested links}

In the second experiment, the previous simulation is run again with same parameters except a slight variation in the network properties mainly for simulating congestion.

Speed. The maximum speed that BEVs can achieve ranges from $20 \mathrm{~km} / \mathrm{h}$ to $70 \mathrm{~km} / \mathrm{h}$ depending upon the location of the links. The links close to facilities get low speed limits whereas links which are further away from facilities gets higher speed limits. 


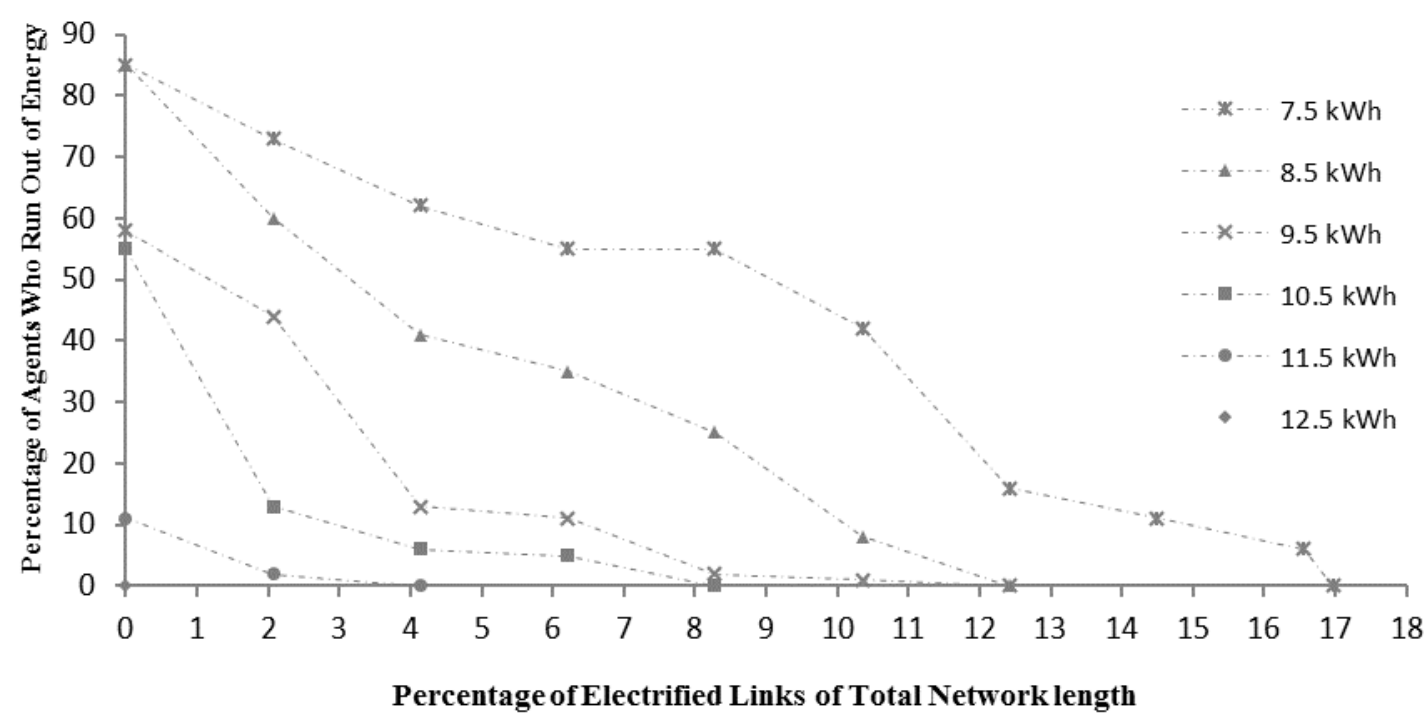

Figure 7. Impact of electrification of the network on different battery sizes

Capacity of the links. The capacity of the links was set high in previous experiment i.e. no congestion was observed. In this experiment the capacity is reduced by taking the traffic count of individual link for an hourly basis from $7 \mathrm{am}-11 \mathrm{pm}$. Later setting their mean values as the capacities of the links. This helps to simulate congestion especially during peak hours. Figure 8 shows the effects of congestion on SOC of three selected agents. In this case the battery size is $7.5 \mathrm{kWh}$ and $19 \%$ of the network is electrified. The graph clearly shows the increase in SOC at different links however, there is a significant increase in the SOC of all agents such that agent 0 at link 15 and 16, agent 33 at link 23 and 24 and agent 65 at link 25 and 26.

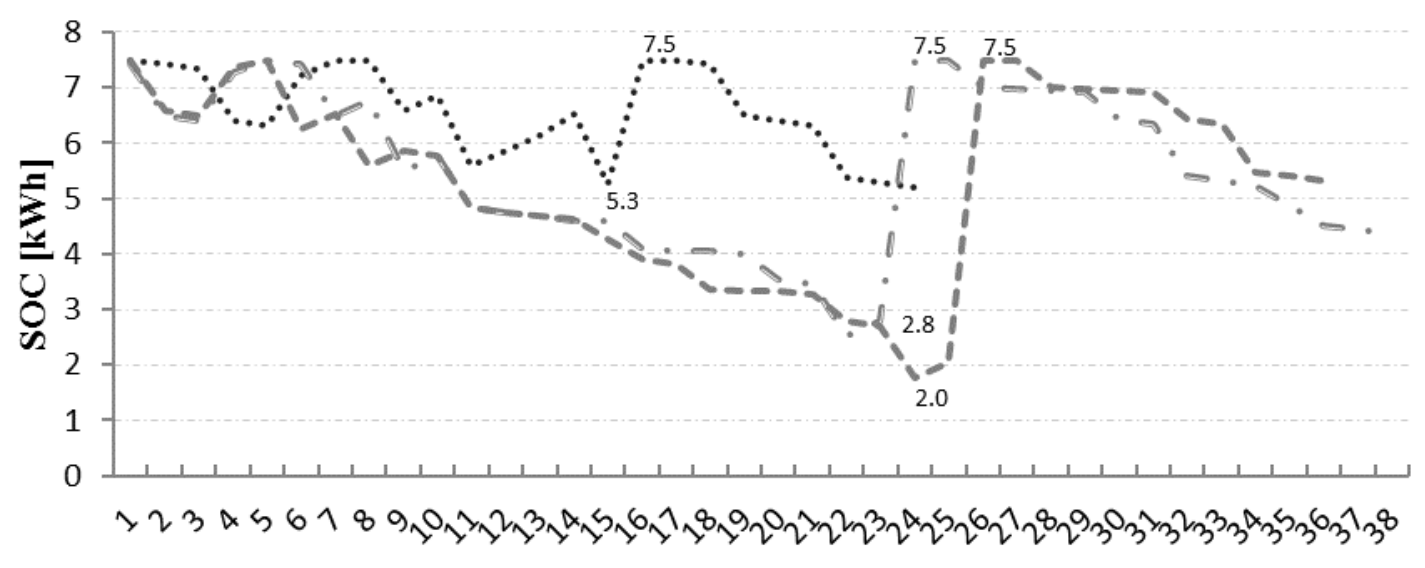

Links of the network

SOC Agent 0 - - SOC Agent $33-\cdots$-SOC Agent 65

Figure 8. Impacts of congestion on SOC of three agents

All of them reach $100 \%$ SOC due to congestion at these links which allows agents to charge longer. As depicted in the graph if agent 33 and agent 65 were not able to charge ca. $5 \mathrm{kWh}$ at congested links both agents' SOC would drop to $0 \%$ before they could reach their final destinations. Moreover, the agents didn't choose to go through congested links 
rather due to change in network configuration they show such behaviour. One can conclude from these results that the inductive charging is ideal for only those sections of the networks which have high traffic counts and have low average speeds.

\section{Electrification of network at road intersections}

In third experiment, the main objective is to analyse inductive charging at intersections. Furthermore, plugin charging is also introduced in the experiment for relative comparison.

Speed. In this experiment same speed limits are used as in experiment two.

Capacity of the links. The capacity of the links is set to high values again. Therefore, there is low traffic congestion in the road network.

Average waiting time at intersection. The traffic light cycle time is distributed evenly to each signal group that means a cycle time is divided into four phase sequence with equal time. The normal cycle time is between 60 to 90 seconds for minor arterial [26]. In this scenario the main objective is to give a general impression about inductive charging at intersections therefore any value can be selected for cycle time between 60 to 90 seconds. A cycle time of 80 seconds is selected which means 20 seconds of signal group for each phase.

The best condition for a vehicle is by facing a green light whenever it arrives at an intersection which means 0 seconds of waiting time and the worst condition is by facing a red light whenever a vehicle arrives at an intersection. In case of red light the maximum waiting time is 60 seconds which is the combined time of other three phases. By taking average of both the best and the worst condition, an average waiting time for stopped vehicles at the intersection is calculated as 30 seconds.

Plugin charging. Workplace facilities are selected for plugin charging where agents can charge their BEVs once they reach workplaces.

Intersections in the network. In the network there are 16 intersections and the network is assumed to be a part of a bigger network. The traffic is considered to be in a steady flow situation.

As shown in Figure 9, agents were able to charge as much as $2 \mathrm{kWh}$ of energy by utilizing their stoppage time at intersections during their everyday activities. This amount of energy is equivalent to ca. $10 \mathrm{~km}$ of driving range extension (assuming $0.2 \mathrm{kWh} / \mathrm{km}$ ). Some agents only charged their vehicles at intersections, as their plans do not contain any work activity where they could charge.

\section{DISCUSSION AND FUTURE WORK}

The work presented in this paper is initial work with regards to simulation of inductive charging of electric vehicles, which is based on the master thesis of the first author [27]. There are many limitations, which we would like to highlight and improve in future.

In this paper all vehicles had the same battery size, based on the energy requirement of the vehicle, which needed the most energy. In future, one could allow different vehicles to have different battery sizes according to their energy needs. Also at the time of this writing, only one type of charging power can be modelled. This is being extended at the 
moment, such that differences in charging capability of different vehicles can be accounted for, as described in [22].

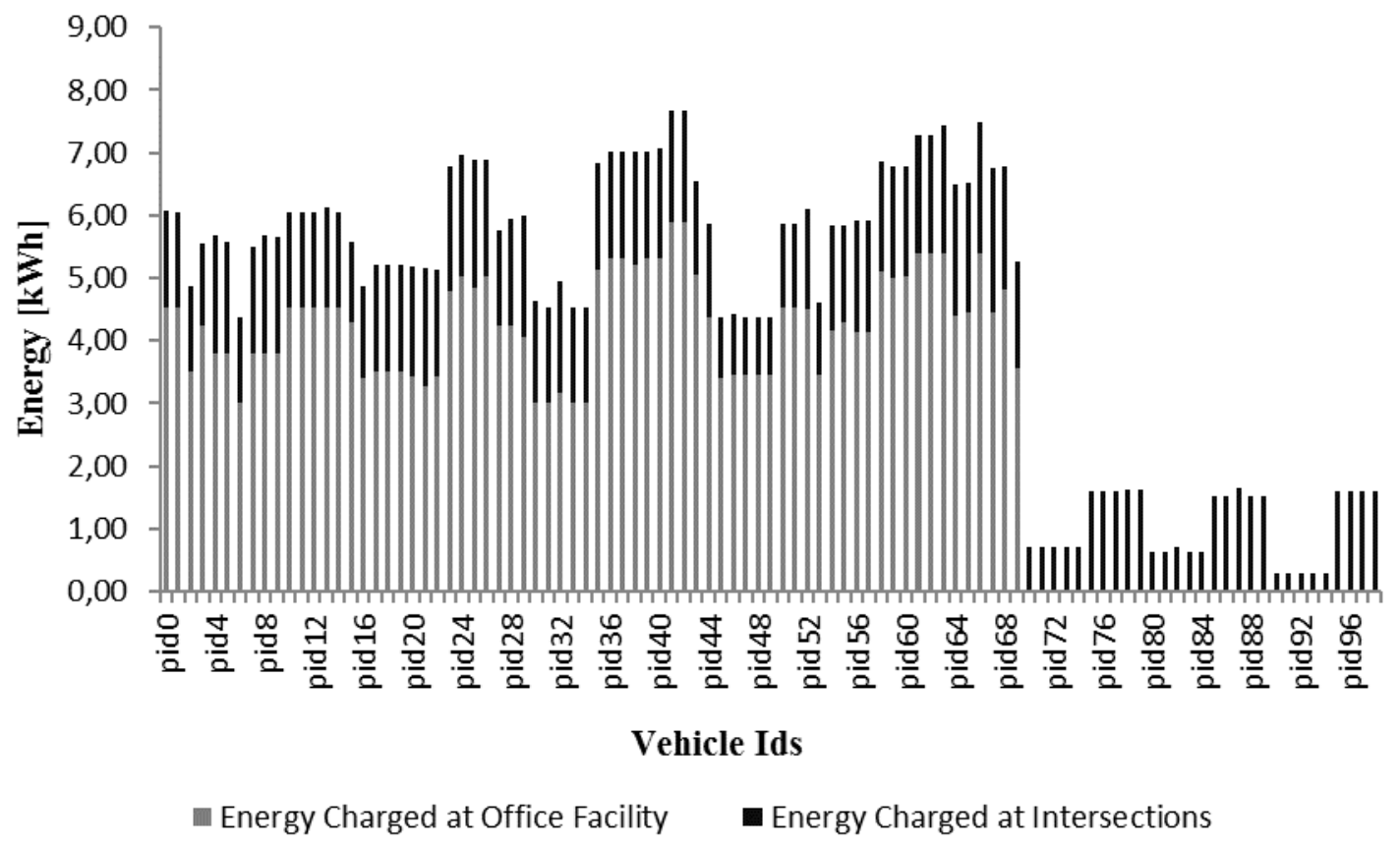

Figure 9. Comparison between energy charged at office facilities and at intersections

In the current version of TES framework, regenerative braking is not modelled. Furthermore, the network used in this paper is two dimensional. In future, TES framework will be extended such that it supports kinetic energy recovery system (KERS) and also allows different energy consumption models for different gradients levels (uphill/downhill) for a more accurate calculation of energy consumption by BEVs. The heuristic for selecting the best road for incorporating inductive charging might also be adapted in future. Instead of using the road, with the highest traffic count, one might also consider the length and average travel time on the road.

Besides these improvements, also work on bigger more realistic scenarios is currently underway where inductive charging for the city of Berlin should be looked at. Also new applications of inductive charging scenarios are being considered: For example for bus based public transit systems one potential location to place an inductive charging system would be at dedicated bus lanes and/or bus stops. Moreover, it is much easier to implement such technology for public transport because the routes of the bus lines are fixed and the demand is known. In this work a cost-benefit analysis is missing, which seems essential to support policy makers and should be incorporated in future. In this case, one might also try out placing inductive charging systems only in certain areas of roads instead of the whole road, e.g. at intersections, where queuing happens.

\section{CONCLUSION}

In this paper, a relatively new charging approach (inductive charging) for BEVs is modelled and simulated successfully by implementing an inductive charging module a framework for electric vehicle simulation. The simulations help to model inductive charging with a detailed spatial and temporal resolution. Different scenarios suggest that inductive charging has a significant effect on battery size of BEVs and is capable of transferring a reasonable amount of energy to BEVs in a short period of time especially in 
case of charging at intersections and as such can support the overall charging infrastructure. In the test scenarios the battery size of BEV could be reduced up to $40 \%$ of its original size by electrifying around $20 \%$ of the total network. This technology can play a complementary role in mass penetration of BEVs.

Besides improving the model further, in future it would be interesting to apply such a model to more realistic scenarios. Such models have been developed for many cities across the globe including Singapore [28].

\section{REFERENCES}

1. International Environment Agency, Key world Energy Statistics 2012, International Environment Agency, Paris, 2012.

2. International Energy Agency, $\mathrm{CO}_{2}$ Emissions from Fuel Combustion Highlights, International Energy Agency, Paris, 2012.

3. Sovacool, B. K. and Hirsh, R. F., Beyond Batteries: An Examination of the Benefits and Barriers to Plug-in Hybrid Electric Vehicles (PHEVs) and a Vehicle-to-grid (V2G) Transition, Energy Policy, vol. 37, no. 3, pp. 1095-1103, 2009., http://dx.doi.org/10.1016/j.enpol.2008.10.005

4. OECD/IEA, Global EV Outlook: Understanding the Electric Vehicle Landscape to 2020, International Energy Agency, Paris, 2013.

5. U.S Dept of Energy, Advanced Vehicles and Fuel: Electric Vehicles, 2010. [Online]. Available: http://www.fueleconomy.gov/feg/evtech.shtml. [Accessed: 14-Feb-2013].

6. EDISON Consortium, Fast Charging of EV Batteries - T at SYSLAB, EDISON, Copenhagen, 2012.

7. National Renewable Energy Laboratory, Plug-In Electric Handbook: For Public Charging Station Hosts, National Renewable Energy Laboratory, 2012.

8. Wu, H. H., Gilchrist, A., Sealy, K., Israelsen, P., Muhs, J., A Review on Inductive Charging for Electric Vehicles, IEEE International Electric Machines \& Drives Conference (IEMDC), Niagara Falls, 2011.

9. Suh, I. S., Application of Shaped Magnetic Field in Resonance (SMFIR), Technology to Future Urban Transportation, Daejeon, 2011.

10. Kobayashi, K., Pontefract, T., Kamiya, Y., Daisho, Y., Development and Performance Evaluation of a Non-contact Rapid Charging Inductive Power Supply System for Electric Micro-bus, Vehicle Power and Propulsion Conference (VPPC), Chicago, 2011.

11. Mohrehkesh, S. and Nadeem, T., Toward a Wireless Charging for Battery Electric Vehicles at Traffic Intersections, Intelligent Transportation Systems (ITSC), Washington, 2011.

12. Jang, Y. J., Ko, Y. D., Jeong, S., Optimal Design of the Wireless Charging Electric Vehicle, Electric Vehicle Conference (IEVC), Greenville, 2012.

13. MATSim, About Us, 2006, http://www.matsim.org/impressum, [Accessed: 20-Oct-2012]

14. Holland, J. H., Adaptation in Natural and Artificial Systems: An Introductory Analysis with Applications to Biology, Control and Artificial Intelligence, Cambridge: MIT Press, 1992.

15. Arnott, R., Palma, A. D., Lindsey, R., A Structural Model of Peak-Period Congestion: A Traffic Bottleneck with Elastic Demand. The American Economic Review, 83(1), pp 161-179, 1993. 
16. Charypar, D. and Nagel, K., Generating Complete All-day Activity Plans with Genetic Algorithms, Transportation, 32(4), pp 396-397, 2005., http://dx.doi.org/10.1007/s11116-004-8287-y

17. Balmer, M., Travel Demand Modelling for Multi-agent Transport Simulations: Algorithms and Systems, PhD. Thesis, Institute for Transport Planning and Systems, ETH, Zurich, 2007.

18. Rieser, M., Adding Transit to an Agent-based Transportation Simulation: Concepts and Implementation, PhD. Thesis, Institute for Transport Planning and Systems, ETH, Zurich, 2010.

19. Galus, M. D., Waraich, R. A., Noembrini, F., Steurs, K., Georges, G., Boulouchos, K., Axhausen, K.W., Andersson, G., Integrating Power Systems, Transport Systems and Vehicle Technology for Electric Mobility Impact Analysis and Efficient Control, IEEE Transactions on Smart Grid, vol. 3, no. 2, pp 934-949, 2012., http://dx.doi.org/10.1109/TSG.2012.2190628

20. Waraich, R. A., Galus, M. D., Dobler, C., Balmer, M., Andersson, G., Axhausen, K. W., Plug-in Hybrid Electric Vehicles and Smart Grids: Investigations Based on a Microsimulation, Transportation Research Part C: Emerging Technologies, vol. 28, pp 74-86, 2013., http://dx.doi.org/10.1016/j.trc.2012.10.011

21. Waraich, R. A., Georges, G., Galus, M. D., Axhausen, K. W., Add-ing Electric Vehicle Modeling Capability to an Agent-based Transport Simulation, In D. Janssens, A. Yasar and L. Knapen (eds.) Data Science and Simulation in Transportation Research, pp 282-318, IGI Global, Hershey, 2014.

22. Waffenschmidt, E., Wireless Power for Mobile Devices, Telecommunications Energy Conference (INTELEC), Amsterdam, 2011.

23. Huh, J. and Rim, C.-T., KAIST Wireless Electric Vehicles- OLEV, Society of Automotive Engineers, 2011.

24. Ahn, S., Suh, N. P., Cho, D.-H., Top 10 Tech Cars, IEEE SPECTRUM, pp 45-50, April 2013..

25. Faria, R., Moura, P., Delgado, J., Almeida, A. T. D., A Sustainability Assessment of Electric Vehicles as a Personal Mobility System, Energy Conversion and Management, vol. 61, pp 19-30, 2012., http://dx.doi.org/10.1016/j.enconman.2012.02.023

26. Koonce, P., Rodegerdts, L., Lee, K., Quayle, S., Beaird, S., Braud, C., Bonneson, J., Tarnoff, P., Urbanik, T., Traffic Signal Timing Manual, 2008.

27. Ul Abedin, Z., Modelling Wireless Charging for Electric Vehicles in MATSim M.Sc. thesis, Dept. Institute of Transportation., Technical University of Munich, Germany, 2012.

28. Ordonez, S., Erath, A., Estimating Dynamic Workplace Capacities Using Public Transport Smart Card Data and a Household Travel Survey, $17^{\text {th }}$ International Conference of Hong Kong Society for Transportation Studies, Hong Kong, 2012. 